die im Magen vor dem Unfall enthalten gewesenen Speisen, vielleicht auch auf ein Blutextravasat bezogen werden können. - Wegen seiner Seltenheit habe ich den Fall veröffentlichen wollen.

$$
\text { Am } \frac{28 \text {. Febr. }}{11 . \text { März }} 1868 \text {. }
$$

3.

\title{
Beobachtungen über congenitalen Defect der grossen Gallengänge und übel Enteritis syphilitica.
}

\author{
Von Dr. M. Roth, \\ Assistenten am pathologischen Institut zu Greifswald.
}

Während meiner Assistenz am pathologischen Institut in Berlin hatte ich Gelegenheit, die zweí folgenden Fälle zu beobehten, die wegen der Seltenheit des Befundes der Veröffentlichung werth scheinen.

\section{Congenitaler Defect der Gallengänge.}

Die Krankheitsgeschichte ergiht über diesen Fall Folgendes:

Albert W., 4 Monate alt, soll mit gelber Hautfarbe geboren und Anfangs ziemlich wohl gewesen, bald aber mager, matt und schlafsüchtig geworden sein. Bei der Aufnahme in die Charité, den 10. October 1867, zeigten sich Conjunct. und die ganze Haut intensiv icterisch, bedeutende Abmagerung. Exantheme, Narben, Oedem nicht vorhanden; am behaarten Kopf und am Nacken mehre kalte Abscesse. Der Tod erfolgte den 30. October.

Von der Anamnese ist nichts bekannt.

Section. Sebr starke Abmagerung. Erheblicher Icterus der Haut und sämmtlicher innerer Organe. Im rechten Umfang des Halses eine kleine oberflächliche Ulceration, unter welcher im subcutanen Gewebe eine umfängliche, mit schleimigem icterischem Eiter gefüllte Höble liegt. Auch über die Ober - und Unterextremităten verbreitet findet sich eine mässige Zahl subcutaner schleimiger Abscesse.

Herz icterisch, an den Klappen nichts Besonderes.

Beide Lungen frei, ziemlich voluminös, icterisch. Im Bereich des vorderen Umfanges beider Lungen eben sichtbare, bis haselnussgrosse, zum Theil netzförmig angeordnete interstitielle Emphysemblasen. Zwischen Ober- und Unterlappen links ein subpleurales frisches Extravasat. Die linke Lunge überall lufthaltig; die rechte im hinteren Umfang von geringen Luftgehalte, von derben hämorrhagischen Stellen durehsetzt. Im Bereich des Mittellappens zahlreiche pleurale miliare Tuberkel, im Parenchym desselben ein etwas über erbsengrosser icterischer Käseknoten mit schiefriger Induration der. Umgebung. Die Lyaphdrüsen am Hilus sehr vergrössert, in derbe käsige Massen verwandelt. 
Die Pleura diaphragmat. zeigt rechts zablreiche Miliartuberkel. Aebnliche Euptionen und zwar in regelmässigen Reihen dem Rjppenver]auf folgend finden sich auf der Pleura costalis dextra.

Milz um mehr als das Doppelte vergrössert, dunkel braunroth, ziemlich derb, von sparsamen, wenig scharf umschriebenen, bis erbsengrossen graurothen Stellen durchsetzt, welche eine weichere Consistenz besitzen.

Beide Nieren mit dïnner leicht trennbarer Kapsel, sehr stark icterisch, hlutarm.

Im Magen wenig bräunlicher, im Duodenum farbloser Schleim.

Leber gross, lederartig derb; Kapsel diffus verdickt, glatt; Oberfläche und Durchschnitt von olivengrüner Farbe; letzterer g]att, blutarm, das stark verdickte graue interstitielle Gewebe setzt sich scharf von der olivengrünen Drüsensnbstanz ab. Die Gallengänge erweitert, der Durchschnitt ist so gefallen, dass er unmittelbar über der Porta auf einen sehr erweiterten, mit grünlichen lrümligen Massen gefüllten, fast 1 Zoll langen Gallengang trifft, der sich nicht auf die Unterläcbe der Leber verfolgen lässt (Anfang des Duct. hepat.).

An Stelle der Gallenblase findet sich ein bloss rabenfederkieldicker, den vorderen Leberrand nicht erreichender Strang, der zwei durch derbes Bindegewebe von einander getrennte erbsengrosse, mit klarer Flüssigkeit gefüllte Cysten enthält. Ein Uebergang in den Duct, cystic. oder auch nur ein diesem entsprechender solider Strang ist nicht vorhanden, ebensowenig der Duct. choled. und bepat. Die Blutgefässe der Leberunterlläche sind normal, die Art. cystid. fell. offen. Die portalen Lymphdrüsen gross und icterisch.

Im Duodenum ist die Papille des Duct. choled. vorbanden, doch dringt die Sonde nur 2 Linien tief in dieselbe ein.

Im Dünndarm farbloser Inbalt. Schleimhant schmutzig gelblich, die Peyerschen Plaques überall leicht vergrössert, gegen die Klappe grauroth. Im untersten Theile des Hleum sind die submucösen Venen ausserordentlich stark varieös. In Dieldarm wenig Schleim, die Solitärfollikel leicht schiefrig. Die Mesenterialdrüsen gross, markig weich, icterisch, fleckig geröthet.

Die Harnblase enthält icterischen Harn.

Schädel: Vordere Fontanelle gross, der übrige Scbädel ziemlicb dick.

Das Gehirn gross, mit zahlreichen Gyris, von zäher Consistenz. Weisse Substanz sehr blass, nicht icterisch, die graue Substanz von normaler Farbe.

Dieser Fall bietet nach mancher Richtung Interesse, so wenig auch eine sichere Deutung des ganzen Befundes, insbesondere bei dem fast gänzlichen Mangel der Anamnese möglich ist. - Was zunächst die Lungen betrifft, so verdient die um einen käsigen Knoten sich findende auf die rechte Pleura beschränkte Miliartuberculose Erwähnung. Das ganz exquisite interstitielle, an einer Stelle hämorrhagische Emphysem beider Langen erklärt sich wohl aus den angestrengten Inspirationen, die bei der ziemlich ausgedehnten blutigen Infiltration der rechten Lunge nôthig waren. - Am wichtigsten sind die Veränderungen der Leber und ihrer Ausführungsaänge. Dass letztere schon bei der Geburt obliterirt waren, geht aus dem von Anfang an bestehenden Icterus und dem comatösen Wesen des Kiades hervor. Gleichwohl lebie das Kind vier Monate, eine Zeitdauer, die bisher bloss 
in einem von Romberg und Henoch ${ }^{1}$ ) beobachteten Fall (der auch in Bezug auf die gleichzeitige interstitielle Hepatitis analog sich verbält) erreicht wurde, während sonst der Tod meist in den ersten Tagen nach der Geburt erfolgte. Es ist sehr wahrscheinlich, dass eine primäre, in früher Periode des Fötallebens eingetretene Peribepatitis die partielle obliteration der Gallenblase und den vollständigen Schwund der Gallengänge zur Folge batte und sich weiter, der Glisson'schen Kapsel folgend, auch auf die Leber selbst erstreckte. Die dadurch bewirkte Hemmung der Pfortadercirculation spricht sich in den erweiterten Venen der Submucosa des Darmes aus. Der Milztumor kommt hier wie in allen Fällen von Lebercirrhose auf Rechuung einer chronischen Hyperplasie.

Bekanntlich ist Perihepatitis und diffuse interstitielle Hepatitis eine häufige Begleiterin congenitaler Syphilis; nehmen wir dazu die tiefen Hautabscesse und die mikroskopisch hier vorgefundene ausgedebnte Fettmetamorphose der Gliazellen des Gehirns, so ist es gewiss erlaubt, auch diesmal die Leberveränderung auf Syphilis zu heziehen, wobei dann der Defect der Gallengänge (dass sie wirklich gebildet waren, beweist die noch vorbandene Gallenblase und die Ausmündungsstelle des Duct. choled.) als Accidens, bedingt durch die Perihepatitis der Leberunterfläche, anzusehen wäre.

Jedenfalls datirt die Veränderung der Leber ziemlich weit in das fötale Leben zurück, da die Cirrhose sehr vorgeschritten ist und die ausführenden Gallengänge auch nicht in Spuren mehr vorhanden sind.

\section{Enteritis sypbilitica.}

Section den 11. März 1868.

Schwach entwickeltes Kind mit blasser Haut.

Der Nabel durch theerartiges Blut etwas vorgetrieben; Nabelgefässe sämmtlich offen, leer, zeigen in der dem Nabel zunächst liegenden Partie schmutzig gelhe Farbe und leichte Verdicknng ihrer Wandungen, am ausgesprochensten die rechte Nabelarterie. Diese enthält an dieser Stelle auch eisen $\frac{1}{2}$ Zoll langen schmutzig graurothen Thrombus.

Die Lungen grösstentheils lufthaltig, nur im Unterlappen der rechten Seite sparsome, über die Schnittfläclıe prominente graubräunliche, ziemlich derbe bronchopneumonische Heerde.

Das. Herz äusserst blass, Klappen unverändert.

Halsorgane blass, normal.

Die Milz gross, prall, Schnitffläche von ziemlich derber Consistenz, sehr blutreich, homogen, Follikel nicht sichtbar.

Die Nebennieren bieten nichts Besonderes.

Linke Niere mit leicht trennbarer Kapsel, mit ausgezeichnetem Harnsăureinfarct der Pyramiden, sonst blass, von normaler Consistenz, ebenso die recite Niere.

Die Schleimbaut des Magens blass, ebenso die des ganzen übrigen Darmtractus,

1) Die hieher gehörige Literatur s. bei Binz, dieses Arch. Bd. XXXV. S. 360 . Leider sind die meisten Fălle nur dürftig beschrieben; für congenitale Syphilis gibt keiner Anhaltspunkte. 
der Inhalt ist dünn bellgelb. Im unteren Theil des Jejunum sind die Solitärfollikel und Peyerschen Plaques etwas prominent, zum Theil von leicht schiefriger Farbe; nahe dem Ileum ist die Serosa zweier benachbarter Darmstücke (das dazwischen liegende Stück beträgt, in gerader Linie gemessen, etwa 2 Zoll) in der Grösse eines Zweigroschensiücks fest verlöthet und zeigt auf den entsprechenden Theilen des Darmlumens je eine geschwürige Stełle. Die höberliegende, mit der Längsachse in der Richtung der Querfalten verlaufend, bohnengross, und auf der concaven Seite des Darmes sitzend, bat in der Mitte einen flachen, unregelmässig begrenzten, etwa erbsengrossen, theils hellgelben, theils dunkelbraunen Schorf von derber Consistenz; um denselben zieht sich eine scbmale, leicht schiefrige, allmäblich in die normale Schleimbaut übergehende, in nächster Nähe des. Schorfes bis auf die Musculatur greifende Verscbwärungszone. Eine linsengrosse Stelle greift sebr tief und nimmt sich wie pine Perforation 3us, doch gelangt die Sonde nicht in das adhärente Darmstück. Das zweite Gescbwïr rundlich, etwa silbersectsergross, sitzt anf dem convexen Unfang des Darmes und betrifft zum Theil einen Peyerschen Plaque (dessen erbaltenes Ende wenig prominent und etwas schiefrig erscheint), überragt denselben seitlich und ist ganz von einem tiefgreifenden gelben Schorf bedeckt, der sich scharf von der etwas anfgeworfenen blassen umgebenden Schleimlaat absetzt. Dasselbe sitzt im untersten Ende des Jejunum: unmittelbar daranf feblen die Kerkring'schen Falten. - Auch im Ileum, besonders ïber der Klappe, prominiren die Plaques etwas; im oberen Theil desselben ist ein zolllanger Plaque in $\frac{2}{3}$ seiner Breite geschwollen, das eine Drittel ist narbenartig glatt und zeigt auf dem Durchschnitt eine flache, die Mucosa und Submucosa betreffende derbe weisse Verdickung. Eine ähnliche narbige Abglättung von Erbsengrösse, zum Theil einen Plaque, zum Theil die anliegende Schleimhaut betreffend, findet sich etwas weiter nach unten, endlich ein dritter Plaque von Erbsengrösse, 3 Zoll über der KIappe, ist in seiner Totalität von dieser Veränderang betroflen.

Die Schleimbaut des Dickdarms blass; im Colon transv. nahe der Flex. col dextr. findet sich ein runder, etwas über groschengrosser, das Nivean leicht überragender Schorf yon derber trockner Beschaffenheit, oberfä̈chlich mit Galle jmbibirt, der in einem Drittel seines Umfanges glatt in die normale Darmschleimbaut übergeht, sonst durch eine schmale in die Submucosa reichende Demarcationszone scharf von derselben abgegrenzt erscheint.

Mesenterialdrïsen, hesonders des lleocöcalstranges, leicht vergrössert, Schnittfläche blassgrau, Consistenz ziemlich derb.

Harnblasenschleimhaut blass.

Leber blutarm, von schmutzig hraungelber Farbe, mit unregelmässigen erbsengrossen, verwaschenen, helleren Stellen, Zejchnung der Acini undeutlich, Consistenz nicht vermehrt.

Schädeldach auffallend dolichocephal, sehr schmal, vordere Fontanelle sehr gross. Auf der Innenfläche der Dura mater, entsprechend den Tubera frontalia, findet sich jederseits eine nach innen flach prominente, mit einem feinen Gefässkranze umgebene, über thalergrosse, weiche, bis $1 \frac{1}{2}$ Linien betragende Verdickung der Dura, die links sich bis auf den seitlichen äusseren Umfang des Orbitaldaches erstreckt. Die Dura ist an diesen Stellen leichter vom Knochen trennbar als 
sonstwo, nimmt, je năher dem Knochen, um so mehr eine weiche fast schleimige Consistenz an und lässt nach der Abtrennung am Knochen eine fein-, stellenweise grobporöse Oberfäche. - Die Innenfläclse der linksseitigen Plaque ist mit der Pia leicht verwachsen, welche eine ganz analoge gelbliche, zweigroschengrosse, nicht auf das Gehirn übergreifende Verdickung zeigte. Eine dritte analoge Stelle von etwa Bohnengrösse findet sich in der linken mittleren Schädelgrube auf dem Felsenbein nahe der Pyramidenspitze. - Die Pia ist zart, trocken, die grossen Gefässe blutarm.

Gehirn von ziemlich guter Consistenz, die graue Substanz äusserst blass, die weisse blutarm, nur in den die Seitenventrikel umgebenden Partien zeigt sich eine diffuse rosige Färbung.

Am äusseren unteren Umfang des linken Femur 1) findet sich ein groschengrosser bis auf den Knochen reichender Heerd, ganz entsprechend den rom Schädel beschriebenen Stellen. Auf dem Durchschnitt verhält sich die Knochensubstanz vollkommen normal.

Die übrigen Extremitätenknochen zeigen keine analogen Veränderungen.

Die Deutung dieser verschiedenen Befunde ist nicht ganz einfach, um so mehr, als auch die Anamnese und Erankengeschichte, die ich Herru Stabsarzt H orn verdanke, keine sicheren Anhaltspunkte bietet. Die Mutter des Kindes, 31 Jahre alt, ist ohne Sparen vou Syphilis, wenn nicht startses Ausgehen der Haxe darauf zu beziehen ist, hat früher ein $5 \frac{1}{2}$ Monat und ein 7 Monat altes Kind geboren, will darauf längere Zeit am Unterleib (?) krank gewesen sein. Das in Rede stehende Kind ist ausgetragen, saugt nicht, liegt mit geschlossenen Angen, öfnet bei Reizen wie Anblasen stets nur das linke Auge, das rechte bleibt geschlossen; Pupillen gleich. Der Mund scheint beim Schreien etwas nach rechts verzogen, überhaupt sind auf dieser Seite die Gesichtsfalten etwas deutlicher ausgesprochen. Mitunter hat es den Anschein, als beständen leicht lösliche Contracturen beider Oberextremitäten. Athmung und Defäcation normal. Tod nach 5 Tagen.

Die auffallendsten und seltensten Veränderungen gehören dem Dünn- und Dickdarm an, und es fragt sich, wean wir zunächst von den narbigen Indurationen einiger Plaques ahsehen, welche Bedeutung den grossen und tiefgreifenden Verschorfungen zukommt. Der zuerst aufgetauchte Gedanke, dass es sich um eine typhöse Erkrankung handle, musste aus sehr verschiedenen Gründen aufgegeben werden. Nicht etwa als ob wir den Typhus bei Neugebornen und ohne dass die Mutter selbst daran erkrankt ist, für eine Unmöglichkeit hielten; sind doch solche wenn auch nicht unzweifelhafte Fälle in der Literatur verzeichnet und wissen wir mit Bestimmtbeit, dass acute Exantheme in dieser Art auftreten können, - es sind zwingende anatomische Gründe, die eine solche Erklärung unmöglich erscheinen lassen. Für's Erste baben die typhösen Veränderungen ihren Sitz wesentlich in den Iymphatischen Apparaten, wenn sie auch häufg genug auf die benachbarte follikelfreie Schleimhaut übergreifen; das zuerst beschriebene Geschwür aber sitzt auf der dem Mesenterium entsprechenden Seite des Darmes, also da wo gar keine Follikel vorkommen. Dazu kammt ferner die Spärlichkeit und die eigenthümliche

1) Präp. No. 2598 der Berliner Sammlung. 
Verbreitung der Ulcerationen, wie dies wobl bei Typhus nie beobachtet wird, dann der Mangel jedweder eigentlich markigen Schwellung der Plaques nnd der Mesenterialdrüsen. Endlich musste schon die selbst bei einem Erwachsenen unerhörte Grösse des Dickdarmgeschwärs für sich allein die höchsten Bedenken gegen eine solche Erkläruag erregen. Vielmehr spricht Alles mehr für eine syphilitische und zwar für eine gummöse Erkrankung der Darmschleimhat, wie sie meines Wissens bisher nicht beschrieben jst "). Zwar kennt man aus reichlichen Fällen bei Erwachsenen die syphilitischen Geschwüre des Dickdarms, weit weniger die des Dünndarms, allein es handelt sich um Erwachsene und immer um vollkommen gereinigte oder vernarbte Geschwüre, deren Genese eben desbalb aicht mehr zu eruiren ist; dagegen haben wir hier wohl anzweifelhaft eimmal das der Geschwürsbildang rorausgebende Stadium, das noch erhaltene, aber schon käsig zerfallene Gummiinfiltrat, das durch die gallige Imbibition und die Demarcationslinie binlänglich als nekrotische Masse charaklerisirt ist. Natürlich ergibt somit auch das Mikroskop keinen charakteristischen Befund.

Neben den specifischen Producten der Syphilis weist der Darm an einigen Peyer'schen Plaques, zum Theil auch an der benachbarten Schleimhaut narbige Zustände der Mucosa und Submucosa auf, die bei mikroskopischer Untersuchung sich folgendermaassen verhalten: auf einem senkrechten Schnitt sieht man zunächst über der ganzen Partie die Zotten fehlen (daher das Glatte und Glänzende dieser Stellen), die oberflächliche Schleimhautschichte van adenoidem Habitus, aber wegen des dicht stehenden Reticulum nicht auspinselbar, die Schleimdrüsen fehlen vollkommen. Viel auffallender ist die Veränderung der tieferen Schichte der Mucosa und der ganzen Submucosa, welche zusammen ein dichtes Flechtwerk mattglänzender sclerotischer Bindegewebsfasern bilden. Durch Essigsäure quellen diese wenig auf und lassen spindel- und sternförmige Zellen hervortreten. Darin eingebettet liegen Follikel oder vielmehr Körper, welche durch Grösse, Form und Anordnung Follikeln entsprechen. Mikroskopisch sieht man in denselben auf Essigsäurezusatz eine grosse Zahl um einen oder mehrere Gefässdurchschnitte concentrisch geordnete spindelförmige Kerne auftreten, von Reticulum and Lymphkörperchen ist keine Spur vorhanden. Die Affection ist demnach als interstitielle Induration mit Verödung der Lymphfollikel aufzufassen, ob mit vorangehender oberflächlicher Geschwärsbildung (Mangel der Zotten), ist zweifelhaft. Auch diese Affection, zusammengehalten mit dem äbrigen Befund, darf wohl unbedingt auf Rechnung der syphilis gesetzt werden. Förster (Würzb. med. Zeitschr. IV. S. 8) bat in einem Fall von congenitaler Syphilis Aebnliches beobachtet und als fibroide Degeneration ler Peyer'schen Drüsen beschrieben.

Unzweifelhaft als gummöse Periostitis zu deuten sind die am Schädel und am inken Femur vorgefundenen gelben Plaques. Nicht nur dass gerade die Stirnhöcker

1) Die von Eberth (dieses Arch. Bd. XL. S. 327) beschriebene gummöse Affection des Dünndarms bei einem Neugebornen betrifft, wenn ich den Verf. recht verstehe, die tieferen Schichten der Darmwandung, während die Mucosa darüber nur "stellenweise gelockert und ulcerirt ist". - Dagegen scheint eine Beobachtung von Bednar (Krankh. d. Neugeb. u. Säugl. Bd. IV. S. 235) bierher zu gebören. 
öfter diese Verănderung bei constatirter congenitaler Syphilis zeigen (Fälle von Howita, Cruveilhier, Charrier), auch der mikroskopisahe Befund weist mit Entschiedenheit darauf hin. Alle Heerde enthalten in reichlicherem oder sparsamerem lackigen Bindegewebe infiltrirt eine grosse Zahl mehr oder weniger verfetteter Lymphkörperchen, grosse Körnchenzellẹn und freie Fettkörnchen. Weicher Natur die massenhaft dazwischen liegenden kleineren nad grösseren prismatischen und nalefformigen lirystalle waren, konnte nicht eruirt werden; Fethrystalle schienen sie nicht za sein. Die Intercellularsubstanz gibt besonders in den dem Knochen oächsten Partien auf Essigsäure und Alkohol reichliche Mucingerinnungen.

Die weisse Substanz des Gehirns zeigte eine Unzahl von Körnchenzellen nicht nur in den gerötheten, sondern auch in den blassen Theilen.

In den derben Hepatisationen der Lunge finden sich neben gewöhnlichen katarrhalischen Zellen sehr viel Körnchenzellen, die zum. Theil diffus hellgelb bis intensiv braungelb gefärbt sind.

Sehen wir, um eine Gesammtliagnose zu gewinnen, ab von einigen unwesentlichen Befunden, so bleiben uns eine gewisse Zahl von Veränderungen, die nach unseren jetzigen Kenatnissen nur auf Syphilis bezogen werden können: so die gummösen Affectionen der Knochen sowie die theils indurativen, theils gummösen Zustände im Darmkanal. Dass die difuse Encephalitis rorwiegend bei congenitaler Syphilis gefunden wird, ist hekannt genug.

Das gefleckte Aussehen der Leber, dies möge hier noch bemerkt werden, berubte in diesem Fall nicht auf partiellen interstitiellen Wucherungen, wie dies ofter bei Syphilis beobachtet wird, sondern lediglich auf verschiedener Blutvertbeilung.

\section{XXII.}

Auszüge und Besprechungen.

1.

J. Kalmus, Die Trichinose in Brünn (Verhandl. des naturf. Vereins in Brünn. 1867. Bd. V. S. 173).

Verf. berichtet über eine Reihe von Erkrankungen an Trichinose in Brünn. von denen einzelne schon früher durch Boner (Allg. Wiener med. Zeilg. 1866 No.6) and Brechler (Wiener med. Presse 1866. No.7) mitgetheilt worden sind Dieselben kamen im Januar 1866 vor und betrafen 8 Personen, darunter zwe Kinder von 10 und 8 Jahren, welche sämmtlich von dem Fleische desselben Schwej nes gegessen hatten. Zweì Erwachsene starben, eine Frau an einem perforirende Duodenal-Geschwür, nachdem jedoch schwere Zufälle der Trichinose vorhergegange waren. Das Sehwein war ungarischer Raçe, jung auf dem Markt gekauft und sei dem im Stalle gefüttert; während der ganzen Zeit seiner Mästung hatte es $n$ 